Pacific Journal of Mathematics

GENERALIZED LERCH ZETA FUNCTION 


\title{
GENERALIZED LERCH ZETA FUNCTION
}

\author{
B. R. JOHNSON
}

The purpose of this paper is to establish certain properties of the generalized Lerch zeta function $\theta(z, \nu, a, b)=$ $\sum_{n=0}^{\infty}(n+a)^{-\nu} z^{(n+a)^{b}}$. The main result yields another infinite series representation for $\theta$. A generalization of Hardy's relation follows as an immediate corollary.

1. Introduction. The function $\Phi(z, \nu, a)$ defined by the power series

$$
\Phi(z, \nu, a)=\sum_{n=0}^{\infty}(n+a)^{-\nu} z^{n},
$$

for $|z|<1,0<a \leqq 1$ and arbitrary $\nu$, is called Lerch's zeta function. For $z=1$, this function becomes Hurwitz' zeta function

$$
\Phi(1, \nu, a)=\zeta(\nu, a)=\sum_{n=0}^{\infty}(n+a)^{-\nu}, \operatorname{Re} \nu>1 \text { and } 0<a \leqq 1 .
$$

Lerch's function has been extensively investigated in [1], [2], [3], [5, v. 1, p. 27], [7], [8], and [12]. One important result

$$
\Phi(z, \nu, a)=\Gamma(1-\nu) z^{-a}(\log 1 / z)^{\nu-1}+z^{-a} \sum_{r=0}^{\infty} \zeta(\nu-r, a) \frac{(\log z)^{r}}{r !},
$$

for $|\log z|<2 \pi, 0<a \leqq 1, \nu \neq 1,2,3, \cdots$, which transforms Lerch's series into another series, is derived in Erdélyi [5, v. 1, p. 29] by using Lerch's transformation formula and Hurwitz' series for the Hurwitz zeta function. Hardy's relation (see Hardy [7] and Mellin [10]) follows immediately from (3):

(4) $\lim _{z \rightarrow 1}\left\{\Phi(z, \nu, a)-\Gamma(1-\nu)(\log 1 / z)^{\nu-1} z^{-a}\right\}=\zeta(\nu, \alpha)$.

The purpose of this paper is to establish certain properties of the function $\theta(z, \nu, a, b)$ where

$$
\theta(z, \nu, a, b)=\sum_{n=0}^{\infty}(n+a)^{-\nu} z^{(n+a)^{b}}, \text { for }|z|<1,0<a \leqq 1,0<b
$$

It is appropriate to call $\theta$ the generalized Lerch zeta function because

$$
\theta(z, \nu, a, 1)=z^{a} \Phi(z, \nu, \alpha)
$$

Using an approach which is more direct than the above mentioned derivation of equation (3), we will establish 


$$
\begin{aligned}
& \theta(z, \nu, a, b) \\
& \quad=b^{-1} \Gamma\left(\frac{1-\nu}{b}\right)(\log 1 / z)^{(\nu-1) / b}+\sum_{r=0}^{\infty} \zeta(\nu-b r, a) \frac{(\log z)^{r}}{r !},
\end{aligned}
$$

where $\nu \neq 1,1+b, 1+2 b, \cdots, 0<a, b \leqq 1$. Formula (6) is valid for unrestricted $z$ if $0<b<1$, and for $|\log z|<2 \pi$ if $b=1$. In the latter case equation (6) becomes equation (3). Furthermore, from (6) we immediately obtain the following generalization of Hardy's relation:

$$
\lim _{z \rightarrow 1}\left\{\theta(z, \nu, a, b)-b^{-1} \Gamma\left(\frac{1-\nu}{b}\right)(\log 1 / z)^{(\nu-1) / b}\right\}=\zeta(\nu, a),
$$

for $0<b \leqq 1$.

2. Derivation of formula (6). Consider the function

$$
f(a)=x^{-\nu} e^{-\beta x^{b}}, \quad \operatorname{Re} \beta>0, b>0 .
$$

The Mellin transform of $f(x)$ with respect to the parameter $s$ is

$$
g(s)=b^{-1} \beta^{(\nu-s) / b} \Gamma\left(\frac{s-\nu}{b}\right), \quad \operatorname{Re} s>\operatorname{Re} \nu, \operatorname{Re} \beta>0, b>0 .
$$

For Mellin transform theory see [4, v. 1, Ch. 2], [10], [11], and [13, p. 46]; for tables see [6, v. 1, p. 303]. Writing $f(x)$ in its Mellin inversion integral form with $x=n+a$, we obtain by summing on $n$ and interchanging the order of summation and integration

$$
\sum_{n=0}^{\infty}(n+\alpha)^{-\nu} e^{-\beta(n+a)^{b}}=\frac{\beta^{\nu / b}}{2 \pi i b} \int_{\sigma_{0}-\infty \infty}^{\sigma_{0}+i \infty} \beta^{-s / b} \zeta(s, a) \Gamma\left(\frac{s-\nu}{b}\right) d s,
$$

where $\sigma_{0}>\max \{1, \operatorname{Re} \nu\}$. The left hand side of (8) is $\theta\left(e^{-\beta}, \nu, a, b\right)$. To evaluate the right hand side integral we will use the residue theorem.

If we denote

$$
h(s)=\beta^{-s / b} \zeta(s, a) \Gamma\left(\frac{s-\nu}{b}\right),
$$

then $h(s)$ has a first order pole at $s=1$ with residue $\beta^{-1 / b} \Gamma([1-\nu / b])$, and an infinite set of first order poles at $s=\nu-b r$ with residues

$$
\frac{(-1)^{r}}{r !} b \beta^{r-2 / b} \zeta(\nu-b r, a), \quad r=0,1,2, \cdots .
$$

Consider the contour integral

$$
\int_{C} h(s) d s,
$$


where the path of integration $C$ is indicated in Figure 1 below, such that the half-circle $C^{\prime}$ of radius $d$ separates the poles $s=\nu-N b$ and $s=\nu-(N+1) b$. Then $h(s)$ is one-valued and analytic inside and on $C$ except at the points $s=1, s=\nu-r b(r=0,1,2, \cdots, N)$.

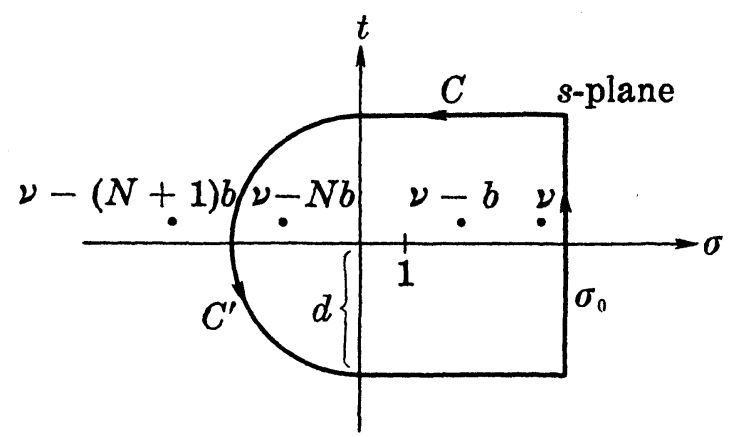

FIG. 1

Now we let $N$ tend to infinity through positive integers.

To investigate the contributions along individual segments of the contour $C$ we will need the following well-known properties of the gamma function and Hurwitz' zeta function, which can be found in Erdélyi [5] and/or Whittaker and Watson [14]:

$$
\begin{gathered}
\Gamma(s)=(2 \pi / s)^{1 / 2} e^{-s} e^{s \log s}\left(1+O\left(\frac{1}{s}\right)\right) \text { as }|s| \longrightarrow \infty,|\arg s|<\pi \\
\frac{\Gamma(s+\alpha)}{\Gamma(s+\beta)}=s^{\alpha-\beta}\left(1+O\left(\frac{1}{s}\right)\right) \text { as }|s| \longrightarrow \infty,|\arg s|<\pi . \\
|\Gamma(\sigma+i t)|=O\left(|t|^{\sigma-1 / 2} e^{-\pi|t| / 2}\right) \text { as }|t| \longrightarrow \infty \\
\text { with } \sigma \text { fixed }(\sigma, t \text { real }) .
\end{gathered}
$$

$$
\zeta(s, a)=2(2 \pi)^{s-1} \Gamma(1-s) \sum_{n=1}^{\infty} n^{s-1} \sin \left(2 \pi n a+\frac{\pi s}{2}\right),
$$

$\operatorname{Re} s<0,0<a \leqq 1$.

$$
\zeta(\sigma+i t, a)=o(|t|) \text { as }|t| \longrightarrow \infty \text { with } 0 \leqq \sigma \text { fixed }(\sigma, t \text { real })
$$

It is clear that the contributions to the integral (10) along the horizontal lines of length $\sigma_{0}$ (see Figure 1 ) vanish as $d \rightarrow \infty$ because of (13) and (15). To find the contribution along the half-circle $C^{\prime}$, it is sufficient to investigate the behavior of $h(s)$ on the quartercircle of radius $d$ for $\pi / 2<\arg s=\phi<\pi$, since the modulus of $h(s)$ on the quarter-circle for $-\pi<\phi<-\pi / 2$ is the same by Schwarz's reflection principle. From (11), (12), and (14) we obtain 


$$
\begin{aligned}
h(s)= & O\left\{|s|^{-\nu / b} e^{|s| \cos \phi\left(\log 2 \pi e-b^{-1} \log \beta e\right)}\right. \\
& \left.\times e^{|s| \sin \phi(\pi / 2-\dot{\phi} \mid b)} e^{|s| \cos \dot{\phi}\left(b^{-1} \log (|s| \mid b)-\log |s|\right)}\right\} \\
& \quad \text { as }|s| \longrightarrow \infty \text { in } \frac{\pi}{2}<\dot{\phi}<\pi, s=|s| e^{i \circ} .
\end{aligned}
$$

In formula (16) we have assumed $\beta$ to be real and positive. The analytic continuation to complex $\beta$ will be obtained later. The following three cases are possible:

(i) $b>1$ : Then (16) is dominated by the last exponential function and $h(s)$ tends to infinity when $d \rightarrow \infty$. Thus, the contribution over the semi-circle tends to infinity as $d \rightarrow \infty$ and formula (8) is not applicable, although the series in (8) converges for $\operatorname{Re} \beta>0$.

(ii) $b=1$ : It is clear from (16) that the integral over the semi-circle $C^{\prime}$ vanishes as $d \rightarrow \infty$, provided $\beta<2 \pi$.

(iii) $0<b<1$ : The integral over the semi-circle $C^{\prime}$ vanishes as $d \rightarrow \infty$, regardless of $\beta$.

Hence, in cases (ii) and (iii) we obtain by (8) and the residue theorem

$$
\theta\left(e^{-\beta}, \nu, a, b\right)-\frac{1}{b} \Gamma\left(\frac{1-\nu}{b}\right) \beta^{(\nu-1) / b}=\sum_{r=0}^{\infty} \frac{(-1)^{r}}{r !} \zeta(\nu-b r, a) \beta^{r} .
$$

The r.h.s. series in (17) is a Taylor series around the origin and is therefore an analytic function of $\beta$ in its circle of convergence, while the l.h.s. expression is valid only when $\operatorname{Re} \beta>0$ and $\nu$ arbitrary; or $\operatorname{Re} \beta=0, \operatorname{Im} \beta \neq 0$, and $\operatorname{Re} \nu>0 ;$ or $\beta=0$ and $\operatorname{Re} \nu>1$. Therefore, (17) represents the analytic continuation with respect to $\beta$ of the l.h.s. of (17) valid for $\operatorname{Re} \beta>0$ into the r.h.s. which is valid for unrestricted $\beta$ when $0<b<1$, or for $|\beta|<2 \pi$ when $b=$ 1. If $b>1,(17)$ is not valid.

Formula (6) is obtained from (17) by setting

$$
z=e^{-\beta}, \quad \beta=\log 1 / z .
$$

ACKNowledgment. The author wishes to thank Professor F. Oberhettinger for his help.

\section{REFERENCES}

1. T. M. Apostol, On the Lerch zeta function, Pacific J. Math., 1 (1951), 161-167.

2. - Remark on the Hurwitz zeta function, Proc. Amer. Math. Soc., 2 (1951), 690-693.

3. E. W. Barnes, On certain functions defined by Taylor series of finite radius of convergence, Proc. London Math. Soc., 4 (1906), 284-316.

4. G. Doetsch, Handbuch der Laplace Transformation, 3 vols., Birkhauser, Basel, $1950-1956$.

5. A. Erdélyi et al., Higher Transcendental Functions, vol. 1, McGraw Hill, New York, 1953. 
6. A. Erdélyi et al., Tables of Integral Transforms, 2 vols., McGraw Hill, New York, 1954.

7. G. H. Hardy, Method for determining the behavior of certain classes of power series near a singular point on the circle of convergence, Proc. London Math. Soc., 3 (1905), 381-389.

8. M. Lerch, Note sur la fonction $\sum_{k=0}^{\infty} e^{2 \pi i k x}(w+k)^{-s}$, Acta Math., 11 (1887), 19-24.

9. G. G. MacFarlane, The application of Mellin transforms to the summation of slowly convergent series, Philos. Mag., (7) 40 (1949), 188-197.

10. H. Mellin, Abriss einer einheitlichen theorie der gamma und der hypergeometrischen funktionen, Math. Ann., 68 (1910), 305-337.

11. - Über den zusammenhang zwischen den linearen differentialund differenzen-gleichungen, Acta Math., 25 (1902), 139-164.

12. F. Oberhettinger, Note on the Lerch zeta function, Pacific J. Math., 6 (1956), $117-120$.

13. E. C. Titchmarsh, Introduction to the Theory of Fourier Integrals, Clarendon, Oxford, 1937.

14. E. T. Whittaker and G. N. Watson, A course in Modern Analysis, 4th ed., Cambridge University Press, London, 1927.

Received July 25, 1973. Research supported in part by National Research Council of Canada under grant number A8199.

UNIVERSITY OF VICTORIA 



\section{PACIFIC JOURNAL OF MATHEMATICS}

\section{EDITORS}

RICHARD ARENS (Managing Editor)

University of California

Los Angeles, California 90024

R. A. Beaumont

University of Washington

Seattle, Washington 98105
J. DugundJI

Department of Mathematics

University of Southern California

Los Angeles, California 90007

D. Gilbarg and J. Milgram

Stanford University

Stanford, California 94305

\section{ASSOCIATE EDITORS}

E. F. BECKENBACH

B. H. NeUMANN

F. WOLF

K. YosHIDA

\section{SUPPORTING INSTITUTIONS}

UNIVERSITY OF BRITISH COLUMBIA

CALIFORNIA INSTITUTE OF TECHNOLOGY

UNIVERSITY OF CALIFORNIA

MONTANA STATE UNIVERSITY

UNIVERSITY OF NEVADA

NEW MEXICO STATE UNIVERSITY

OREGON STATE UNIVERSITY

UNIVERSITY OF OREGON

OSAKA UNIVERSITY
UNIVERSITY OF SOUTHERN CALIFORNIA

STANFORD UNIVERSITY

UNIVERSITY OF TOKYO

UNIVERSITY OF UTAH

WASHINGTON STATE UNIVERSITY

UNIVERSITY OF WASHINGTON

$\stackrel{*}{*} \stackrel{*}{*} \stackrel{*}{*}$ AMERICAN MATHEMATICAL SOCIETY




\section{Pacific Journal of Mathematics}

\section{Vol. 53, No. $1 \quad$ March, 1974}

Martin Bartelt, Strongly unique best approximates to a function on a set, and a finite

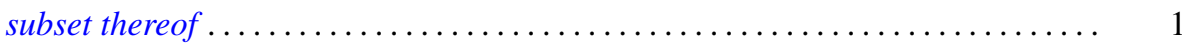

S. J. Bernau, Theorems of Korovkin type for $L_{p}$-spaces $\ldots \ldots \ldots \ldots \ldots \ldots \ldots \ldots \ldots$

S. J. Bernau and Howard E. Lacey, The range of a contractive projection on an

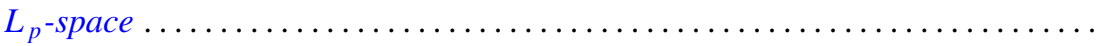

Marilyn Breen, Decomposition theorems for 3-convex subsets of the plane ......... Ronald Elroy Bruck, Jr., A common fixed point theorem for a commuting family of

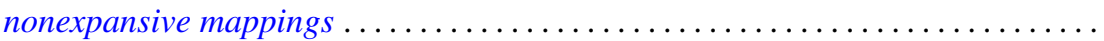

Aiden A. Bruen and J. C. Fisher, Blocking sets and complete $k$-arcs . . . . . . . 73

R. Creighton Buck, Approximation properties of vector valued functions . ......... 85

Mary Rodriguez Embry and Marvin Rosenblum, Spectra, tensor products, and

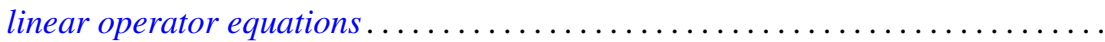

Edward William Formanek, Maximal quotient rings of group rings . . . . . . . . . 109

Barry J. Gardner, Some aspects of T-nilpotence . . . . . . . . . . . . . . . 117

Juan A. Gatica and William A. Kirk, A fixed point theorem for $k$-set-contractions

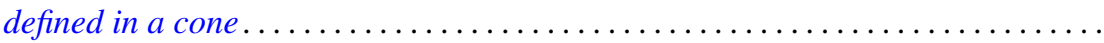

Kenneth R. Goodearl, Localization and splitting in hereditary noetherian prime

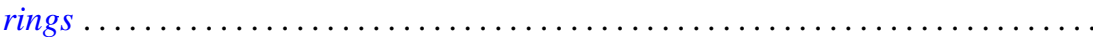

James Victor Herod, Generators for evolution systems with quasi continuous

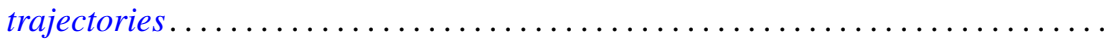

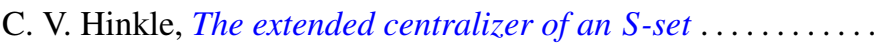

I. Martin (Irving) Isaacs, Lifting Brauer characters of p-solvable groups . . .

Bruce R. Johnson, Generalized Lerch zeta function ...........

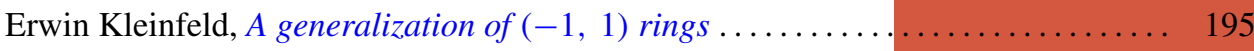

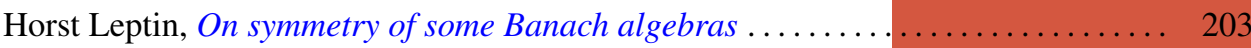

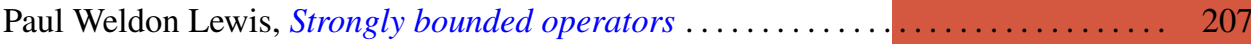

Arthur Larry Lieberman, Spectral distribution of the sum of self-adjoint

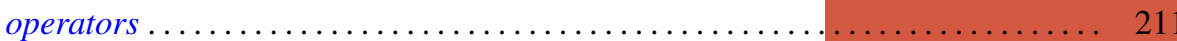

I. J. Maddox and Michael A. L. Willey, Continuous operators on paranormed spaces and matrix transformations

James Dolan Reid, On rings on groups ........................... 229

Richard Miles Schori and James Edward West, Hyperspaces of graphs are Hilbert cubes.

William H. Specht, A factorization theorem for p-constrained groups ...

Robert L Thele, Iterative techniques for approximation of fixed points of certain nonlinear mappings in Banach spaces ...............

Tim Eden Traynor, An elementary proof of the lifting theorem

Charles Irvin Vinsonhaler and William Jennings Wickless, Completely decomposable groups which admit only nilpotent multiplications .

Raymond O’Neil Wells, Jr, Comparison of de Rham and Dolbeault cohomology for

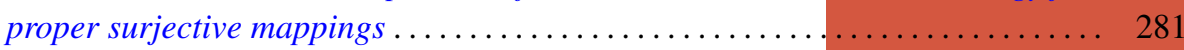

David Lee Wright, The non-minimality of induced central representations . . . . . 301 